\title{
Weight Management Outcomes of Youth with Autism Spectrum Disorder Seeking Treatment from a Multidisciplinary Team
}

\author{
Haley J. Killian ${ }^{1} \cdot$ Isabella K. Pallotto ${ }^{2} \cdot$ Brooke R. Sweeney $^{3} \cdot$ Meredith L. Dreyer Gillette $^{4}$ \\ Accepted: 16 March 2021 / Published online: 31 March 2021 \\ (c) The Author(s), under exclusive licence to Springer Science+Business Media, LLC, part of Springer Nature 2021
}

\begin{abstract}
Youth with Autism Spectrum Disorder (ASD) are at an increased risk for developing obesity when compared to their typically developing peers. Given higher prevalence of obesity in youth with ASD, understanding factors relating to success in obesity treatment provides insight into implementing efficacious treatments for youth. The current study examines age, sleep, and metabolic factors potentially affecting success in 74 youth $\left(M_{\text {age }}=11.66\right)$ attending a multidisciplinary weight management treatment program over a year. Multilevel modeling indicated that higher baseline BMI class category, medications at baseline, and absence of sleep difficulties predicted greater reduction in BMI after a year of treatment.
\end{abstract}

Keywords Pediatric weight management $\cdot$ Autism $\cdot$ Obesity treatment $\cdot$ Weight management outcomes

\section{Introduction}

Obesity in youth is associated with poorer mental health and concurrent and future health risks such as cardiovascular disease, diabetes, and cancer (CDC 2020). One

Meredith L. Dreyer Gillette

mldreyer@cmh.edu

Haley J. Killian

haleykillian@mail.umkc.edu

Isabella K. Pallotto

ispallotto@unc.edu

Brooke R. Sweeney

brsweeney@cmh.edu

1 Department of Psychology, The University of Missouri Kansas City, 5030 Cherry St, Kansas City, MO 64110, USA

2 Department of Health Behavior, Gillings School of Global Public Health, The University of North Carolina at Chapel Hill, 135 Dauer Dr, Chapel Hill, NC 27599, USA

3 Department of Pediatrics, Weight Management and Center for Children's Healthy Lifestyles \& Nutrition, Children's Mercy Kansas City/University of Missouri Kansas City School of Medicine, 2401 Gillham Rd., Kansas City, MO 64108, USA

4 Department of Pediatrics, Developmental and Behavioral Health and Center for Children's Healthy Lifestyles \& Nutrition, Children's Mercy Kansas City/University of Missouri Kansas City School of Medicine, 2401 Gillham Rd., Kansas City, MO 64108, USA subgroup demonstrated to be at particular risk for obesity is youth with Autism Spectrum Disorder (ASD) (Curtin et al. 2005; Grondhuis \& Aman, 2014; Healy et al. 2019). In the US, approximately 1 in 54 youth have a diagnosis of ASD (Maenner et al. 2020), and previous research suggests that the prevalence of overweight and obesity in youth with ASD is significantly higher at younger ages ( $2-5$ years of age) and in adolescence (12-17 years) (Hill et al. 2015). While the exact cause of this trend is unknown, research suggests youth with ASD have a greater number of obesogenic risk factors than their typically developing (TD) peers (Coury et al. 2012; Must, Curtin, et al., 2014; Sharp et al. 2013) including medications that promote weight gain and risk for metabolic syndrome (Maayan \& Correll, 2011; Seida et al. 2012), increased sedentary behavior (Dreyer Gillette et al. 2015; Must, Phillips, et al., 2014), feeding difficulties (Ledford \& Gast, 2006; Marshall et al. 2014), and sleep problems (Elrod $\&$ Hood, 2015).

Due to the potential for developing adverse health conditions, it is essential to develop efficacious weight management programs that address the unique needs of youth with ASD. Behavioral modifications suggested to TD youth, such as increasing physical activity and healthy eating behaviors (Dreyer Gillette et al. 2015), need to be tailored to youth with ASD's specific needs. Weight management clinics that provide customized treatment targeting specific problematic eating behaviors commonly seen in youth with ASD and developmental disabilities led to 
significant reductions in BMI (Dreyer Gillette et al. 2014; Pona et al. 2017). Results showed that younger children in the program had a greater decrease in BMI z-score (a standardized measure of body mass index) (Pona et al. 2017). The purpose of the present study was to evaluate whether a multidisciplinary weight management treatment program targeted for youth with special needs, such as ASD, was effective in producing sustained reductions in youth BMI among youth diagnosed with ASD. Additionally, we aimed to see whether baseline metabolic parameters, medication status, and demographic variables predicted more successful weight loss in these youth seeking weight management treatment.

The present study sought to understand the relationship between child factors, such as sleep, baseline BMI category, and baseline metabolic parameters, and how those factors predicted child BMI change during a weight management treatment program designed specifically for youth with ASD over a one year-period. In this study, obesity is defined as having a BMI greater than the 95th percentile based on age and gender norms. Given the wide age range of our population, BMI percent of the 95th percentile (BMI\%95th) was used to standardize the data analysis across time and to account for the severity of obesity. To assist with mapping child BMI onto adult BMI classifications, the CDC has proposed BMI classifications based on the BMI \%95th (class 1 obesity: BMI $\geq 95$ th percentile- $120 \%$ of the 95 th percentile; class 2 severe obesity BMI $\geq 120 \%$ to $140 \%$ of the 95th percentile; class 3 severe obesity BMI $\geq 140 \%$ of the 95th percentile) (Skinner et al. 2015).

Several a priori hypotheses were tested. (1) Younger youth with ASD will experience greater reductions in BMI\%95th following a year of treatment, as suggested in previous literature in weight management treatment within a group of youth with developmental and physical difficulties (Pona et al. 2017). (2) BMI class at baseline will predict weight loss over the course of the year, such that those with class 1 obesity who initiate treatment would experience the largest reduction in BMI\%95th. (3) Youth with baseline behavioral and medication risk factors at baseline (e.g., medications known to impact appetite, sleep problems) will experience smaller BMI\%95th change over one year. Evaluation of factors relating to the success of weight management treatment programs is important for the utilization of efficacious treatment modalities when treating youth with obesity, specifically those with ASD. The current study sought to evaluate 12-month outcomes of youth with ASD enrolled in special needs specific pediatric weight management treatment and understand the factors that may positively influence treatment success, defined as a reduction in BMI\%95th. It also provides an illustration of youth with ASD who are seeking weight management treatment from a specialized multidisciplinary team dedicated to treating obesity in youth with physical and developmental disabilities.

\section{Method}

\section{Participants}

Participants consisted of youth $(N=74)$ who presented within the last 5 years for multidisciplinary obesity treatment from the Special Needs Weight Management Clinic at a large hospital in the Midwest. Youth were between the ages of 2 and 18 years of age, had a BMI $\geq 95$ th percentile based on age and gender norms, had attended at least 2 clinic visits over the course of a year, and spoke either English or Spanish as their primary language. While the clinic serves children with diverse special healthcare needs, we focused the present sample solely on those with a documented diagnosis of ASD, which was recorded in the electronic medical record (EMR) by the clinic physician or psychologist. Youth were excluded from the analysis if they had only attended one clinic visit as it was assumed that they were not actively participating in weight management treatment. Additionally, 1 participant was excluded due to the clinic visit at 12 months occurring at the beginning of COVID-19 pandemic and an accurate weight could not be obtained to calculate BMI for analyses.

Youth were referred to the clinic by primary care physicians and subspecialists (e.g., sleep medicine, endocrinology, cardiology) for weight management treatment. Families typically attended appointments once every 2-3 months unless medication management was initiated (then approximately monthly appointments were scheduled). Clinic recommendations entailed a combination of dietary (MyPlate, decrease sugared drinks, change snacks, increase protein), environmental/behavioral (stimulus control, meal/snack schedules, sleep hygiene), and medical (diagnose/treat sleep apnea, optimize ADHD care, initiate medication management). Youth and their families were seen in person at the outpatient clinic or at one of 4 telemedicine sites located between $90 \mathrm{~min}$ and $3 \mathrm{~h}$ from the main hospital campus. These telemedicine locations were at hospital clinics and included a trained nurse to collect height and weight and assist in facilitated physical exam with ancillary devices like a stethoscope and camera. See Dreyer Gillette et al. (2014) and Pona et al. (2017) for additional details regarding clinic procedures and treatment. Data collection and research procedures were approved by the hospital's Institutional Review Board. Prior to any data being utilized in the current research project, parents gave consent for clinical outcome data to be utilized for research purposes. 


\section{Measures}

Demographics/Anthropometric/Metabolic/Medication status: Youth demographic variables such as age, sex, and insurance status were extracted from the EMR at baseline. Laboratory values such as cholesterol, liver function tests, and hemoglobin A1c were also extracted from the EMR for baseline data. Height and weight measurements were recorded to the nearest $0.1 \mathrm{~cm} / \mathrm{kg}$ and were completed at each visit by a trained clinical provider (e.g., care assistant or registered nurse). Finally, comorbid diagnoses and medication status were recorded at baseline in the EMR and extracted.

Baseline questionnaire: Parents completed a questionnaire upon enrollment in the program that provided information about the family demographics and asked about parent perceptions of several different challenges that may occur in this population to include sleep problems. These questions were asked in the form of Likert scales and yes/no questions (sleep problems).

\section{Statistical Analysis}

The present study sought to assess the long-term outcomes of weight management within a population of youth with ASD. It also sought to assess outcomes within the Special Needs Weight Management Clinic that differed based on patient characteristics (i.e., age, sex, ethnicity, insurance status, and parent reports of other difficulties). Patients who were enrolled in treatment beginning January of 2014 and who had attended an initial visit and had passed their 12-month follow-up window were considered eligible for inclusion in the analysis. The current study utilized a multilevel model to evaluate change in BMI\%95th score from baseline to 12-month follow-up time points. This model of analysis was utilized due to its unique abilities to include all available data while adjusting to account for missing data. These adjustments include accounting for correlations between repeated measure BMI\%95th of patients, handling missing data without having to utilize multiple imputations, flexibility in modeling both fixed and random effects occurring from time-related variables, and evaluating cross-level interactions between patient-level moderating effects (Enders, 2001).

Due to the attrition evidenced within the clinic (35.1\% did not return for a 12-month follow up appointment), MLM was deemed most appropriate to adequately assess changes while reducing the possibility of bias due to patient dropout. The model included person-level repeated measures of BMI\%95th percentile (level 1) nested within individuals (level 2) and controlled for baseline BMI\%95th percentile and patient characteristics using a random intercepts model and a homoscedastic error structure (i.e., scaled identity).
A variable to examine both 6-month and 12-month since baseline was included in the model to illustrate linear change in BMI\%95th across weight management treatment. This linear variable was squared to create a quadratic change that allows for evaluating accelerated growth rate across treatment. Finally, additional interactions were examined to evaluate whether patient characteristics at baseline influenced the BMI\%95th reduction at 12-months. These variables were explored in relation to the linear time variable while controlling for patient characteristics: age, gender/sex, race/ethnicity, baseline sleep difficulties, Hemoglobin A1c, and medication use.

Statistical analyses were completed using IBM SPSS Statistics (Version 26), predictive analytics software, using the mixed procedure and the full information maximum likelihood estimator. This estimation was utilized, as opposed to the restricted maximum estimation, because it allows for evaluations of fixed effects in nested models, which are the variables of most interest in the current project (Kwok et al. 2008). This type of estimator can adequately handle missing data within a longitudinal analysis when data are missing completely at random on the variables of interest, which is indicated by Little's Missing Completely at Random test $(\chi 2=46.91, p=0.67)$ (Enders, 2001).

\section{Results}

The average patient BMI at baseline was 134.23 BMI\%95th ( $S D=27.69 \mathrm{BMI} \% 95$ th), and the average patient age was 11.66 years $(S D=3.86$ years). Most patients were male $(74.7 \%)$ and identified as Caucasian (62.7\%). Per the EMR, patients had comorbid diagnoses of ADHD (54.2\%), asthma $(18.1 \%)$, and sleep apnea (8.4\%). Of all patients enrolled in the weight management clinic, $21.4 \%$ were taking atypical antipsychotics, and $27.7 \%$ were taking stimulants at baseline (see Table 1). About half of the patients had commercial insurance (50.6\%), and patients visited the weight management clinic, on average, 4.08 times annually (range $=2-8$ visits; see Fig. 1). Upon evaluating differences in patient demographics between those who attended a 12 month follow up and those who did not, only insurance status was significant in that patients with Medicaid were significantly more likely to return for a 12-month follow up than those who had commercial insurance or self-pay, $\left(X^{2}(2)=6.06\right.$, $p=0.048)$.

To evaluate changes in BMI in patients with ASD over the course of a year in treatment, we utilized BMI\%95th. This metric is used to evaluate changes in obesity, particularly for youth who sit at the very high end of weight curves, which allows for BMI comparisons to age and sex-specific BMI percentiles (Flegal \& Cole, 2013; Freedman \& Berenson, 2017). Both linear and quadratic 
Table 1 Descriptive statistics of the sample
Fig. 1 Number of annual visits to the weight management clinic

\begin{tabular}{|c|c|c|c|}
\hline & $M(S D)$ & Minimum-maximum & $n(\%)$ \\
\hline Age (years) & $11.66(3.86)$ & $2.72-18.95$ & \\
\hline Number of visits & $3.97(1.48)$ & $2-8$ & \\
\hline BMI & $32.18(1.48)$ & $19.55-59.82$ & \\
\hline BMI $z$-score & $2.42(0.48)$ & $1.59-4.27$ & \\
\hline BMI \%95th percentile & $134.23(27.69)$ & $98.48-226.06$ & \\
\hline Sex & & & $15(20.3)$ \\
\hline Female & & & $59(79.7)$ \\
\hline \multicolumn{4}{|l|}{ Male } \\
\hline Ethnicity & & & $10(13.5)$ \\
\hline African American/Black & & & $46(62.2)$ \\
\hline Caucasian & & & $11(14.9)$ \\
\hline Latino/a/x & & & $7(9.5)$ \\
\hline \multicolumn{4}{|l|}{ Other } \\
\hline \multicolumn{4}{|l|}{ Comorbid diagnoses } \\
\hline $\mathrm{ADHD}$ & & & $37(50)$ \\
\hline Asthma & & & $14(18.9)$ \\
\hline Sleep apnea & & & $7(9.5)$ \\
\hline \multicolumn{4}{|l|}{ Medications } \\
\hline Atypical antipsychotics & & & $18(24.3)$ \\
\hline Stimulants & & & $20(27.0)$ \\
\hline \multicolumn{4}{|l|}{ Insurance status } \\
\hline Commercial & & & $38(51.4)$ \\
\hline $\begin{array}{l}\text { Medicaid, financial assistance, } \\
\text { or self }\end{array}$ & & & $36(48.6)$ \\
\hline
\end{tabular}

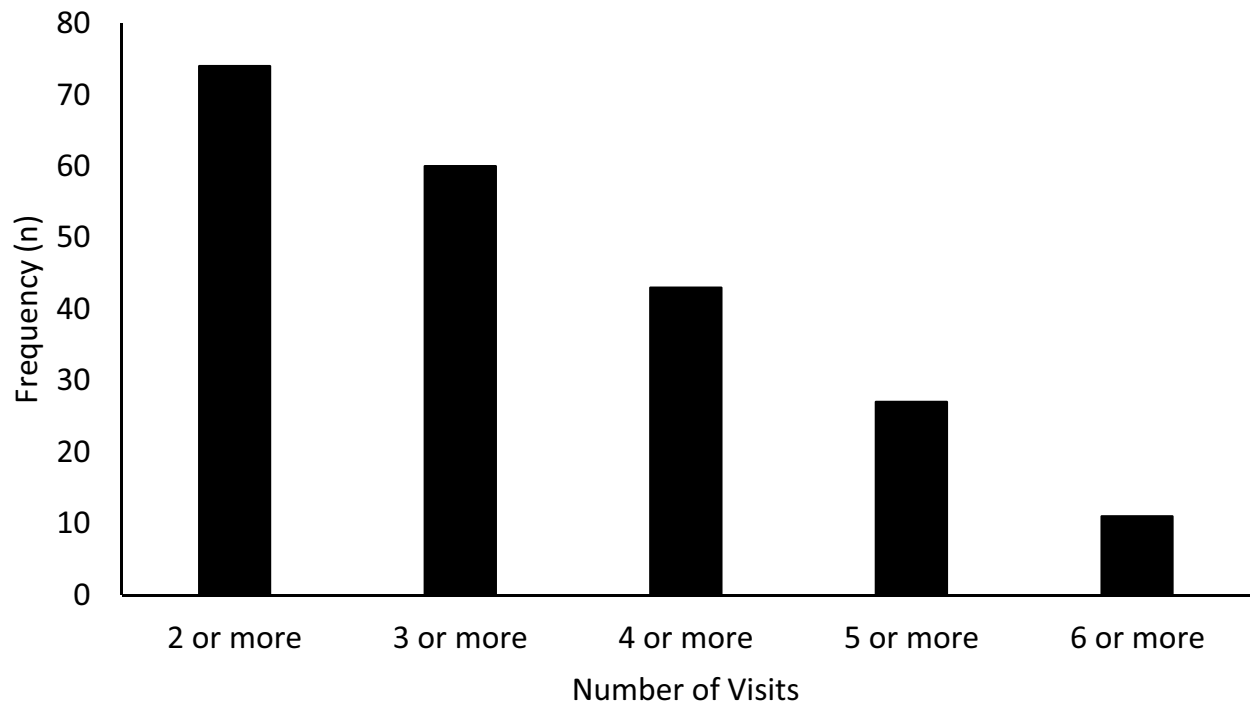

growth rates indicated significant changes in BMI\%95th over the course of a year and thus were retained in the model. The model demonstrated a significant linear decrease in BMI\%95th over a year, $\beta=-8.93$, standard error $(S E)=2.28, t=-3.92$; and $p<0.001$. In addition, the model demonstrated a significant quadratic time variable in explaining change in BMI\%95th over the course of treatment, $\beta=2.25 ; S E=1.12 ; t=2.01$; and $p=0.048$ ). These results taken together indicate a significant reduction in BMI\%95th over the course of a year; with more pronounced weight reduction early in treatment and beginning to plateau over time for youth with ASD in 
specialized multidisciplinary weight management treatment as is typical with weight loss trajectory over time.

When taking patient characteristics into account and examining how they may indicate differential treatment progress over the course of a year (as measured by BMI\%95th), several variables indicated predictive power. The final model included BMI class category at baseline, sleep problems per parent report at baseline, stimulant use, atypical antipsychotic use, and visits to clinic, while controlling for patient characteristics of age, race/ethnicity, and gender/sex. There were significant linear reductions in
BMI\%95th percentile over time (measured at 6-months and then again at 12-months), $(\beta=-8.45, S E=3.21 ; t=-2.63$; and $p=0.010$ ). In addition, there were significant quadratic changes in BMI\%95th percentile over the course of a year $(\beta=2.47, S E=1.01 ; t=2.45$; and $p=0.017)$. The main effect of BMI class category was significant $(\beta=25.40, S E=2.12$; $t=11.97$; and $p<0.001)$. In addition, the interaction between BMI class category at baseline and linear time while controlling for patient characteristics was significant, $(\beta=-1.93$, $S E=0.71 ; t=-2.71$; and $p=0.008)$. This finding suggests that children who were in a higher BMI class category at
Fig. 2 a Reductions in BMI\% of the 95 th over 12 months based on obesity class category. $\mathbf{b}$ Reductions in $\mathrm{BMI} \%$ of the 95 th over 12 months based on parent reported sleep problems

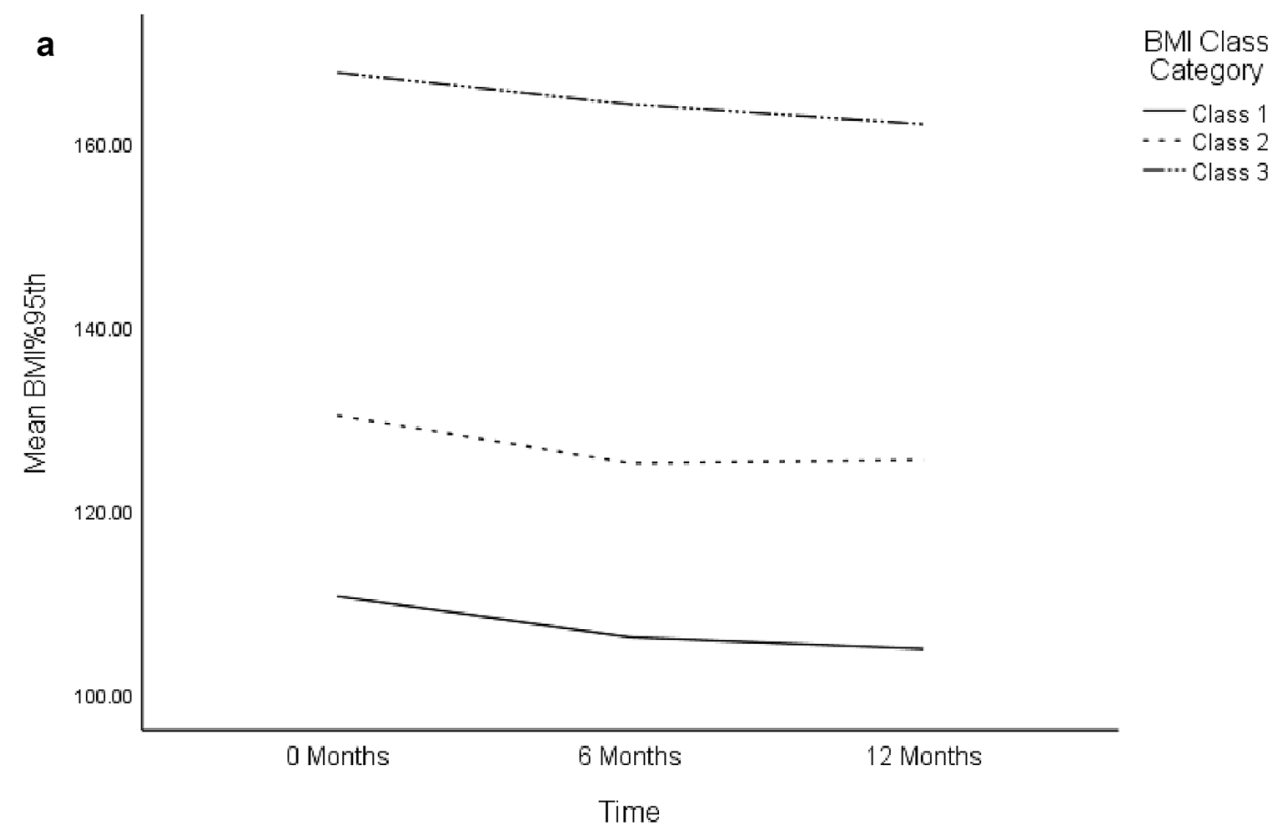

b

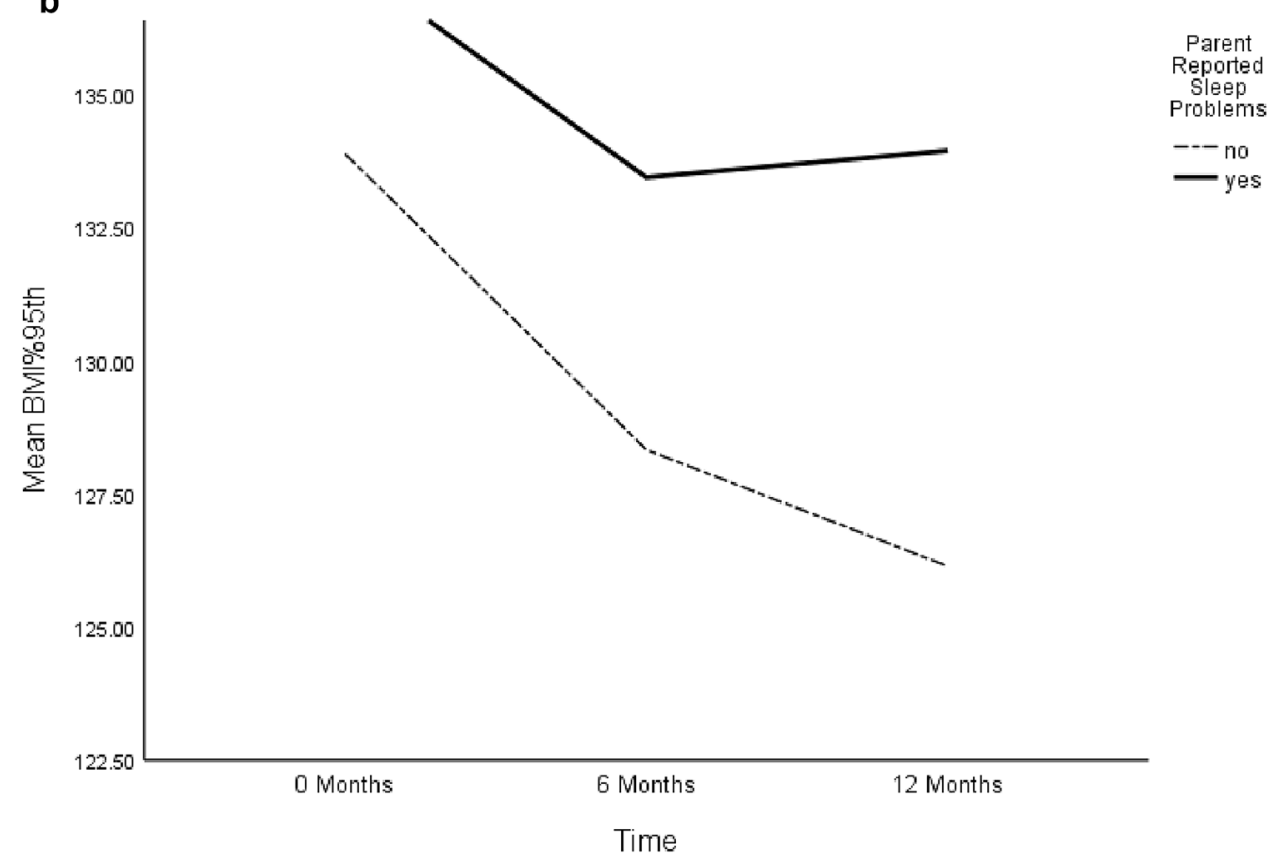


baseline had greater reductions in BMI\%95th percentile over time (See Fig. 2a). The main effect of baseline sleep problems was not significant in predicting BMI\%95th percentile ( $\beta=-0.27, S E=3.29 ; t=-0.08$; and $p=0.94)$; however, the interaction between sleep problems and linear time while controlling for patient characteristics was significant $(\beta=3.87, S E=1.13 ; t=3.42$; and $p=0.001)$ (See Fig. $2 b$ ). This result suggests that children with parent-reported sleep problems had slightly smaller reductions in BMI\%95th percentile when compared to those children whose parents reported no sleep problems at baseline. The main effect of stimulant use was significant in predicting BMI\%95th percentile $(\beta=10.54, S E=3.63 ; t=2.91$; and $p=0.005)$. This result suggests that youth who were taking stimulants at baseline had higher BMI\%95th. The main effect of taking atypical antipsychotic medications at baseline, was not significant in predicting BMI\%95th percentile $(\beta=4.78$, $S E=4.03 ; t=1.19$; and $p=0.24)$; however, the interaction between taking atypical antipsychotic medications at baseline and linear time while controlling for patient characteristics was significant $(\beta=-3.71, S E=1.26 ; t=-2.94$; and $p=0.004)$. This finding suggests that youth who were taking atypical antipsychotic medications at baseline had greater reductions in BMI\%95th over time. The main effect of number of visits was significant in predicting BMI\%95th percentile $(\beta=4.45, S E=1.11 ; t=4.00$; and $p<0.001)$. This result suggests that youth who attend more visits have higher baseline BMI\%95th. Variables including age, race/ethnicity, and gender/sex were not significant predictors of BMI\%95th percentile change in this group of individuals.

\section{Discussion}

The current study presents findings to provide insight on patient factors that contribute to overall reduction in BMI\%95th of youth with ASD who are enrolled in a multidisciplinary, family-based weight management clinic. The results indicate significant linear reductions in BMI\%95th following a year of treatment. When controlling for patient characteristics, these results suggest that BMI class category, stimulant and atypical antipsychotic medications, and parent reported sleep difficulties significantly influence response to a weight loss intervention for these youth. The results of these patient-level factors may provide clinicians treating these youth with important insight on additional factors to consider when creating treatment plans.

\section{BMI Class Category}

Children who have class 3 severe obesity experienced greater reductions in BMI than those youth with class 1 obesity or class 2 severe obesity over the course of treatment. This result was contrary to our hypotheses; however, it is consistent with previous weight management research suggesting that youth with more severe obesity experience greater reductions in weight to approach the mean (Kumar et al. 2019). More severe obesity at baseline would be expected to be treated with more intensive weight loss interventions (e.g., medication management, more frequent visits), often coupled with more concern from families leading to improved readiness to implement changes.

\section{Medications}

When considering the role that medications play in youths' baseline BMI\%95th, or the moderating effect that they have on subsequent weight loss, the current study suggested that both stimulants and atypical antipsychotic medications contribute to a child's BMI\%95th status. Previous literature has attributed weight gain to atypical antipsychotics (Scahill et al. 2016), which would suggest that it may also hinder treatment success within the context of weight management. The results of the current study suggest that youth who are taking atypical antipsychotic medications at baseline experience greater weight loss over the course of a year in weight management treatment. These contrary findings may also suggest that parents are more aware of the problematic eating behaviors related to the hunger from the medications and also know what their child's eating looked like prior to beginning atypical antipsychotic medications. Potential explanations for the unexpected finding include that parents here highly concerned about their child's eating patterns and hunger relative to the medication's side effects and were more motivated to implement treatment recommendations and seek out higher levels of treatment. The multidisciplinary treatment team may also be more aggressive, both from a medication and behavioral standpoint, to address the obesity related to atypical antipsychotic medications. A future direction may be to evaluate whether these youth are more likely to have intensive and advanced treatment such as more frequent clinic visits or be prescribed pharmacotherapy to support weight loss or symptom control, such as topiramate and metformin.

A common side effect associated with stimulant medication use is appetite suppression, which suggests that it might also be associated with decreases in weight in combination with behavioral recommendations suggested by the treatment team (Poulton et al. 2016). Although the current study did not find the effect over time, youth who were on stimulants at baseline had higher BMI\%95th than those who were not taking stimulant medication. Stimulant medication is often used to treat the symptoms associated with attentiondeficit hyperactivity disorder (ADHD) (Coury et al. 2012), and this finding suggests that youths needing stimulant medications may have had higher levels of impulsivity related 
to food seeking or eating behaviors which could contribute to the development of obesity. In the current study, 54.2\% of youth had a comorbid diagnosis of ADHD, and future investigations could qualitatively document the reasons that youths with ASD were placed on stimulant medications.

\section{Number of Visits}

Although the number of visits attended over the course of a year did not moderate the linear weight loss experienced by youth in clinic, it is important to note that youth with higher baseline BMI\%95th attended clinic more frequently than those who had lower baseline BMI\%95th. This suggests that youth identified early on to have higher BMI may need more frequent contact with a weight management team to help assist the family in implementing behavioral or medication recommendations, or are given more intensive treatment plans by clinic practitioners. Youth who are on medications that support weight loss are seen more frequently in this clinic which would result in more visits over the course of a year to allow for adjustments in dosage or evidence a more intensive treatment plan.

\section{Sleep Problems}

Over time, youth with sleep problems experienced a smaller reduction in BMI\%95th percentile compared to their counterparts with no sleep problems. This result suggests that sleep difficulties may be an important area to consider when thinking about effective treatment for weight management with these youth. This result aligns with previous literature suggesting a relationship between short sleep duration and, both, poor diet quality and less frequent physical activity (Hager et al. 2016).

\section{Demographic Factors}

When evaluating changes in BMI\%95th over the course of a year, patient demographics such as gender/sex, age, and race/ethnicity (de Vinck-Baroody et al. 2015) did not moderate or help to predict BMI\%95th percentile change over the course of treatment. However, previous literature suggested that when examining the change in BMIz scores, rather than BMI\%95th, over the course of treatment, age did moderate the BMIz change. This finding suggests that age may play a larger role when examining BMIz scores as opposed to BMI\%95th as BMI\%95th is believed to be less sensitive to age effects (Pona et al. 2017).

When evaluating differences in youth who attended their 12 month visit and those who did not, there were no differences based on demographic variables except for insurance status. Youth who were on Medicaid were more likely to return for a 12-month follow up than those who had commercial insurance or were self-pay. This may be due to the difficulty that families have reported in getting insurance companies to cover the cost of needed weight management treatment (Wilfley et al. 2017). Cost of treatment, when weight management treatment is not covered, may present a real barrier for getting youth with ASD and obesity the treatment they need.

\section{Limitations}

Literature surrounding weight management treatment within a population of youth with developmental disabilities is sparse. One significant limitation of the current study is the relatively small sample size. Although the sample began at 74 patients, after a year of treatment, 48 patients remained enrolled or attended their 12-month follow-up appointment. The rates of attrition, while relatively high, are similar to other studies of participants in weight management after a year of treatment (Savoye et al. 2007). Another limitation is that all patients were treated within one specific clinic at one specific hospital in the current study. Although this represents a limitation, there are relatively few multidisciplinary teams around the United States dedicated to treating youth with obesity and developmental and physical disabilities. Finally, the level of ASD severity was not measured in the current study. This represents a limitation in that it is difficult to know how severity of autism impacted treatment recommendations or ability to implement given recommendations. Some of these youth may have had difficulty achieving weight loss due to difficulty adhering to treatment recommendations or medication nonadherence, associated with their ASD. Although this represents a potential limitation to the current study, past work has examined the relationship between BMI and adaptive functioning and found that the two are unrelated (Egan et al. 2013).

\section{Future Directions}

The current study provides a starting point related to patient characteristics that contribute to success in weight management outcomes for youth with ASD. This presents several considerations for clinicians to incorporate into treatment planning; however, further research is needed to inform clinical interventions for greater treatment success. An additional factor that may be related to weight status is feeding difficulties or problematic mealtime behaviors, which is frequently reported as a difficulty in this group, and youth with ASD experience more frequent feeding difficulties when compared to youth with other developmental disabilities. Future research to strengthen these findings should include adding eating or feeding difficulties into the analytic model. In addition, future research with a larger sample, at multiple 
sites, still using a multidisciplinary treatment team, for youth with ASD could further inform future improvements in treatment. A final future research direction would be to examine whether these BMI\%95th decreases are sustained over a long-term follow up period, which would be an important given that obesity often continues into adulthood.

Acknowledgments The authors wish to thank the Health Care Foundation of Greater Kansas City (now known as the Health Forward Foundation) for funding the development of the program. We also wish to thank our clinical colleagues for their assistance in delivering the intervention and collecting data. As noted in our paper, our current work extends prior work (Dreyer Gillette et al., 2014 and Pona et al., 2017) on clinical outcomes of the entire sample of participants in our clinic for children with special needs by utilizing a focused sample of youth with ASD.

Funding This program in which the study is based in was funded by the Health Care Foundation of Grater Kansas City (now known as the Health Forward Foundation).

\section{Declarations}

Conflict of interest The authors have no conflicts of interest to declare that are relevant to the content of this article.

Research involving human participants and/or animals Approval was obtained from the ethics committee of Children's Mercy Kansas City. The procedures used in this study adhere to the tenets of the Declaration of Helsinki. (Ethics approval number: 11120379).

Informed consent Informed consent was obtained from legal guardians.

Author Contributions All authors contributed to the study conception and design. Material preparation, data collection and analysis were performed by HJK, IKP, BRS, and MLD. The first draft of the manuscript was written by HJK, IKP and MLD and all authors commented on previous versions of the manuscript. All authors read and approved the final manuscript.

\section{References}

Center for Disease Control. (2020). Childhood obesity causes and consequences. https://www.cdc.gov/obesity/childhood/causes.html.

Coury, D. L., Anagnostou, E., Manning-Courtney, P., Reynolds, A., Cole, L., McCoy, R., et al. (2012). Use of psychotropic medication in children and adolescents with autism spectrum disorders. Pediatrics, 130(Supplement 2), S69-S76. https://doi.org/10.1542/ peds.2012-0900D.

Curtin, C., Bandini, L. G., Perrin, E. C., Tybor, D. J., \& Must, A. (2005). Prevalence of overweight in children and adolescents with attention deficit hyperactivity disorder and autism spectrum disorders: a chart review. BMC Pediatrics, 5(1), 48. https://doi.org/ 10.1186/1471-2431-5-48.

de Vinck-Baroody, O., Shui, A., Macklin, E. A., Hyman, S. L., Leventhal, J. M., \& Weitzman, C. (2015). Overweight and obesity in a sample of children with autism spectrum disorder. Academic Pediatrics, 15(4), 396-404.
Dreyer Gillette, M. L., Borner, K. B., Nadler, C. B., Poppert, K. M., Odar Stough, C., Swinburne Romine, R., et al. (2015). Prevalence and health correlates of overweight and obesity in children with autism spectrum disorder. Journal of Developmental \& Behavioral Pediatrics. https://doi.org/10.1097/DBP.0000000000000198.

Dreyer Gillette, M. L., Stough, C. O., Beck, A. R., Maliszewski, G., Best, C. M., Gerling, J. K., et al. (2014). Outcomes of a weight management clinic for children with special needs. Journal of Developmental \& Behavioral Pediatrics, 35(4), 266-273. https://doi.org/10.1097/DBP.0000000000000055.

Egan, A. M., Dreyer, M. L., Odar, C. C., Beckwith, M., \& Garrison, C. B. (2013). Obesity in young children with autism spectrum disorders: Prevalence and associated factors. Childhood Obesity, 9(2), 125-131.

Elrod, M. G., \& Hood, B. S. (2015). Sleep differences among children with Autism Spectrum Disorder. Journal of Developmental \& Behavioral Pediatrics, 36(3), 166-177.

Enders, C. K. (2001). The performance of the full information maximum likelihood estimator in multiple regression models with missing data. Educational and Psychological Measurement, 61(5), 713-740.

Flegal, K. M., \& Cole, T. J. (2013). Construction of LMS parameters for the Centers for Disease Control and Prevention 2000 growth charts, Vol. 63. US Department of Health and Human Services, Centers for Disease Control.

Freedman, D. S., \& Berenson, G. S. (2017). Tracking of BMI z scores for severe obesity. Pediatrics, 140(3), e20171072.

Grondhuis, S. N., \& Aman, M. G. (2014). Overweight and obesity in youth with developmental disabilities: A call to action. Journal of Intellectual Disability Research, 58(9), 787-799. https://doi. org/10.1111/jir.12090.

Hager, E. R., Calamaro, C. J., Bentley, L. M., Hurley, K. M., Wang, Y., \& Black, M. M. (2016). Nighttime sleep duration and sleep behaviors among toddlers from low-income families: associations with obesogenic behaviors and obesity and the role of parenting. Childhood Obesity, 12(5), 392-400.

Healy, S., Aigner, C. J., \& Haegele, J. A. (2019). Prevalence of overweight and obesity among US youth with autism spectrum disorder. Autism, 23(4), 1046-1050.

Hill, A. P., Zuckerman, K. E., \& Fombonne, E. (2015). Obesity and autism. Pediatrics, 136(6), 1051-1061. https://doi.org/10.1542/ peds.2015-1437.

Kumar, S., King, E. C., Christison, A. L., Kelly, A. S., Ariza, A. J., Borzutzky, C., et al. (2019). Health outcomes of youth in clinical pediatric weight management programs in POWER. The Journal of Pediatrics, 208(57-65), e54.

Kwok, O.-M., Underhill, A. T., Berry, J. W., Luo, W., Elliott, T. R., \& Yoon, M. (2008). Analyzing longitudinal data with multilevel models: An example with individuals living with lower extremity intra-articular fractures. Rehabilitation Psychology, 53(3), 370.

Ledford, J. R., \& Gast, D. L. (2006). Feeding problems in children with autism spectrum disorders. Focus on Autism and Other Developmental Disabilities, 21(3), 14.

Maayan, L., \& Correll, C. U. (2011). Weight gain and metabolic risks associated with antipsychotic medications in children and adolescents. Journal of Child and Adolescent Psychopharmacology, 21(6), 517-535. https://doi.org/10.1089/cap.2011.0015.

Maenner, M. J., Shaw, K. A., \& Baio, J. (2020). Prevalence of autism spectrum disorder among children aged 8 years-Autism and developmental disabilities monitoring network, 11 sites, United States, 2016. MMWR Surveillance Summaries, 69(4), 1.

Marshall, J., Hill, R. J., Ziviani, J., \& Dodrill, P. (2014). Features of feeding difficulty in children with autism spectrum disorder. International Journal of Speech-Language Pathology, 16(2), 151-158. https://doi.org/10.3109/17549507.2013.808700. 
Must, A., Curtin, C., Hubbard, K., Sikich, L., Bedford, J., \& Bandini, L. (2014). Obesity prevention for children with developmental disabilities. Current Obesity Reports, 3(2), 156-170.

Must, A., Phillips, S. M., Curtin, C., Anderson, S. E., Maslin, M., Lividini, K., et al. (2014). Comparison of sedentary behaviors between children with autism spectrum disorders and typically developing children. Autism, 18(4), 376-384. https://doi.org/10. 1177/1362361313479039.

Pona, A. A., Dreyer Gillette, M. L., Odar Stough, C., Gerling, J. K., \& Sweeney, B. R. (2017). Long-term outcomes of a multidisciplinary weight management intervention for youth with disabilities. Childhood Obesity, 13(6), 455-461. https://doi.org/10.1089/chi. 2016.0334

Poulton, A. S., Hibbert, E. J., Champion, B. L., \& Nanan, R. K. (2016). Stimulants for the control of hedonic appetite. Frontiers in Pharmacology, 7, 105.

Savoye, M., Shaw, M., Dziura, J., Tamborlane, W. V., Rose, P., Guandalini, C., et al. (2007). Effects of a weight management program on body composition and metabolic parameters in overweight children: A randomized controlled trial. JAMA, 297(24), 2697. https://doi.org/10.1001/jama.297.24.2697.

Scahill, L., Jeon, S., Boorin, S. J., McDougle, C. J., Aman, M. G., Dziura, J., et al. (2016). Weight gain and metabolic consequences of risperidone in young children with autism spectrum disorder. Journal of the American Academy of Child \& Adolescent Psychiatry, 55(5), 415-423. https://doi.org/10.1016/j.jaac.2016.02.016.
Seida, J. C., Schouten, J. R., Boylan, K., Newton, A. S., Mousavi, S. S., Beaith, A., et al. (2012). Antipsychotics for children and young adults: A comparative effectiveness review. Pediatrics, 129(3), e771-e784. https://doi.org/10.1542/peds.2011-2158.

Sharp, W. G., Berry, R. C., McCracken, C., Nuhu, N. N., Marvel, E., Saulnier, C. A., et al. (2013). Feeding problems and nutrient intake in children with Autism Spectrum Disorders: A metaanalysis and comprehensive review of the literature. Journal of Autism and Developmental Disorders, 43(9), 2159-2173. https:// doi.org/10.1007/s10803-013-1771-5.

Skinner, A. C., Perrin, E. M., Moss, L. A., \& Skelton, J. A. (2015). Cardiometabolic risks and severity of obesity in children and young adults. New England Journal of Medicine, 373(14), 1307-1317.

Wilfley, D. E., Staiano, A. E., Altman, M., Lindros, J., Lima, A., Hassink, S. G., \& Improving Access and Systems of Care for Evidence-Based Childhood Obesity Treatment Conference Workgroup. (2017). Improving access and systems of care for evidencebased childhood obesity treatment: Conference key findings and next steps. Obesity, 25(1), 16-29.

Publisher's Note Springer Nature remains neutral with regard to jurisdictional claims in published maps and institutional affiliations. 